\title{
POWER LINE COMMUNICATIONS APLICADO A GENERACIÓN EÓLICA BAJO CONDICIONES CLIMÁTICAS SEVERAS
}

\section{Power Line Communications Applied to Eolic Generation Under Severe Climatic Conditions.}

Presentación: 06/10/2020

\author{
Doctorando:
}

\section{Adriana Laura Ibarreta Fañanas}

Facultad Regional Santa Cruz (FRSC) - Facultad Regional Buenos Aires (FRBA) - Universidad Tecnológica Nacional (UTN) - Argentina libarretaf@frsc.utn.edu.ar

\section{Director/es:}

\section{Mario Blas Lavorato}

Co-director/es:

\section{Resumen}

El propósito de este artículo es presentar una propuesta de plan de tesis y nivel de avance del mismo.

Power Line Communication (PLC) es una técnica ampliamente utilizada, principalmente como medio de comunicación en Smart Grid y redes in-building, sin embargo, en la actualidad se está comenzando a utilizar en zonas rurales o de difícil acceso, similares a las que presenta la Patagonia Austral. A pesar de esto, su utilización en el medio nacional adaptada para este tipo de aplicaciones, no registra muchos antecedentes.

El objetivo del presente plan de trabajo es el estudio de las técnicas y protocolos de comunicación PLC, a ser utilizados en la región austral del país, para la comunicación entre un aerogenerador de eje vertical instalado en una zona remota y una central de comando y control alimentada por el mencionado aerogenerador.

Dadas las condiciones de la región, es necesario conocer los parámetros, variables estructurales y accionamiento del aerogenerador en tiempo real. Por ello se propone modelar y luego implementar un protocolo de comunicación y control apto para la situación planteada.

Palabras clave:, Power Line Communication, Aerogenerador, Condiciones Climáticas Severas, Procesamiento de Señales.

\footnotetext{
Abstract

The main purpose of this article is to present the thesis plan proposal and its advances so far.

Power Line Communicactions (PLC) is a well know technique, principally in Smart Grid communications and inbuilding networks, however, actually its started to be presented as a communication solution for rural zones or areas difficult to access, similar to the ones established in the Southern Patagonia region. Nevertheless, there are no registers about the use of this technique for this application in Argentina.

The objective of the present work is to study of the techniques and communication protocols, to be used in the southern region of this country, to communicate a vertical axis wind generator installed in a remote aera, with a command and control central powered by this wind generator.

Considering the conditions in this region, it's necessary to know the parameters, structural variables and power on and off control of the generator, in real time. Therefore it is proposed to model and implement a communication protocol and control according to the exhibit situation.
} 
Keywords: Power Line Communication, Wind Generator, Severe Climate Conditions, Signal Processing.

\section{Introducción}

La técnica conocida como Power Line Communications (PLC) es una tecnología antigua y ampliamente conocida (Rabie, Adebisi, \& Tonello, 2018) (Han, Jeong, Lee, \& Kim, 2017) (Choi \& Jung, 2017) (Milioudis, Andreou, \& Labridis, 2015), sin embargo, su utilización en el medio nacional adaptada para este tipo de aplicaciones no registra antecedentes.

En la actualidad sus usos son muy variados y dicha técnica está muy asociada al uso de Smart Grid (Prasad \& Lampe, 2020) (López, y otros, 2019), ya que no requiere una instalación específica para la comunicación entre partes, utilizando el mismo tendido eléctrico. Según sus usos puede ser clasificada en tres tipos:

- De acceso (Access PLC) proporcionado por las empresas proveedoras de energía quienes ofrecen internet a través de los cables de baja tensión, transmitiendo entre frecuencias de 1,6 y $40 \mathrm{MHz}$. Muy poco difundida. (Yoon, Jang, Kim, \& Bahk, 2014)

- Para control y transmisión de datos (Control PLC), lo que posteriormente dio lugar a la domótica. Esta tecnología PLC opera debajo de los 500 KHz. (Zhilenkov, Gilyazov, Matveev, \& Krishtal, 2017)

- $\quad$ Para LAN dentro de edificios (in-home PLC) (Tonello, Versolatto, \& Pittolo, 2014). Creada originalmente en Estados Unidos y muy utilizada en Japón, esta tecnología se utiliza para crear una red de alta velocidad dentro de un edificio, a través del tendido eléctrico del mismo. También se han presentado propuestas de aplicarlo en otros recintos cerrados como vehículos (Xu, Yang, Tan, \& Sheng, 2017) (Takanashi, y otros, 2014), aplicaciones navales (Antoniali, Tonello, Lenardon, \& Qualizza, 2011), aeronaves (Dégardin, y otros, 2010) y trenes (Artale, y otros, 2013).

Sin embargo, existen otros usos que aún no han sido masivos para esta técnica. Un ejemplo es la aplicación de PLC para áreas rurales, o zonas remotas. Dado que en estas áreas generalmente las compañías de teléfono o cable no llegan, las señales de radio son pobres y la comunicación satelital es extremadamente costosa, esta técnica puede llegar a ser una gran solución, especialmente en lo que se refiere a mediciones y recopilación de información de sensores.

En nuestro país, hay grandes zonas semidesérticas - entre ellas la región Patagónica - donde existen comunidades del tipo aisladas, con condiciones climáticas muy duras, pero que a diferencia de las demás, posee un gran potencial para la generación de energía eléctrica (Bahamonde, Samela, Szewzuk, \& Ibarreta Fañanas, 2013). Sin embargo, por las razones del tipo socio-económicas y geográficas, los grandes parques eólicos se concentran cercanos al sistema interconectado nacional, relegando nuevamente las zonas rurales, conformadas por pequeñas poblaciones de bajos recursos y geográficamente aisladas.

Las condiciones climáticas de la Patagonia Austral, no solo contemplan las conocidas bajas temperaturas en invierno, y la presencia de escarcha desde el otoño, sino también la constante presencia de vientos fuertes, de carácter rafagoso, pudiendo arrastrar partículas y pequeñas rocas capaces de dañar cualquier estructura mecánica. Es dificultosa la utilización de aerogeneradores de eje horizontal comerciales debido a que no están fabricados para estas condiciones climáticas, ya que las velocidades promedio de vientos de esta zona, son similares a las velocidades máximas que éstos soportan. Sumado a esto, se necesitan para su monitoreo y control, estructuras capaces de soportar estas condiciones donde montar los sistemas de comunicaciones que permitan vincular al operador con el aerogenerador.

Por esta razón es que el presente plan de tesis propone la utilización de la técnica PLC para realizar un monitoreo y control de parada, en tiempo real, de un aerogenerador de eje vertical, destinado a zonas aisladas de la Patagonia Austral. En particular, se pretende presentar un protocolo de comunicación, capaz de realizar el deseado envío de datos bilateral, teniendo en cuenta los retos que este escenario presenta.

\section{Desarrollo}

Para el Desarrollo del presente plan de tesis, se realizó una amplia búsqueda bibliográfica. En primer lugar, se realizó un estudio de las condiciones climáticas, estado energético de la Patagonia Austral y posibles aerogeneradores que puedan soportar las mencionadas exigencias. Dado que los aerogeneradores de tipo comercial están diseñados 
para detenerse a velocidades que son similares a la media de vientos presentes en la provincia de Santa Cruz, se decidió por adoptar un diseño de eje vertical, que para su funcionamiento óptimo no requiere ser instalado a gran altura, y cuya instalación no perjudique las características de la vivienda a la cual se destina el mencionado aerogenerador, asegurando un menor impacto.

Por otro lado, se realizó la búsqueda bibliográfica de un posible juego de sensores necesarios para conocer el estado del aerogenerador en tiempo real, de modo de poder conocer la información mínima que debería contener el paquete de datos a enviarse por la conexión eléctrica entre el aerogenerador y la vivienda. Para este caso se contemplaron sensores de temperatura, velocidad (del eje y de las palas), vibraciones, presión y deformación para la estructura.

En esta materia, se realizó un pequeño estudio del estado del arte, de cada tipo de sensor para poder tener conocimiento de con qué nuevas herramientas se puede contar a la hora de seleccionar los sensores a utilizar.

Posteriormente, una vez cubiertos los temas básicos se realizó un estudio acerca de la técnica a utilizar (Power Line Communication) teniendo en cuenta los retos que ya de por sí esta técnica trae aparejados, por utilizar como canal de comunicación el mismo conductor que energiza la vivienda, destinada a ser receptor y posterior transmisor de la información. El sistema propuesto se muestra en la Figura 1.

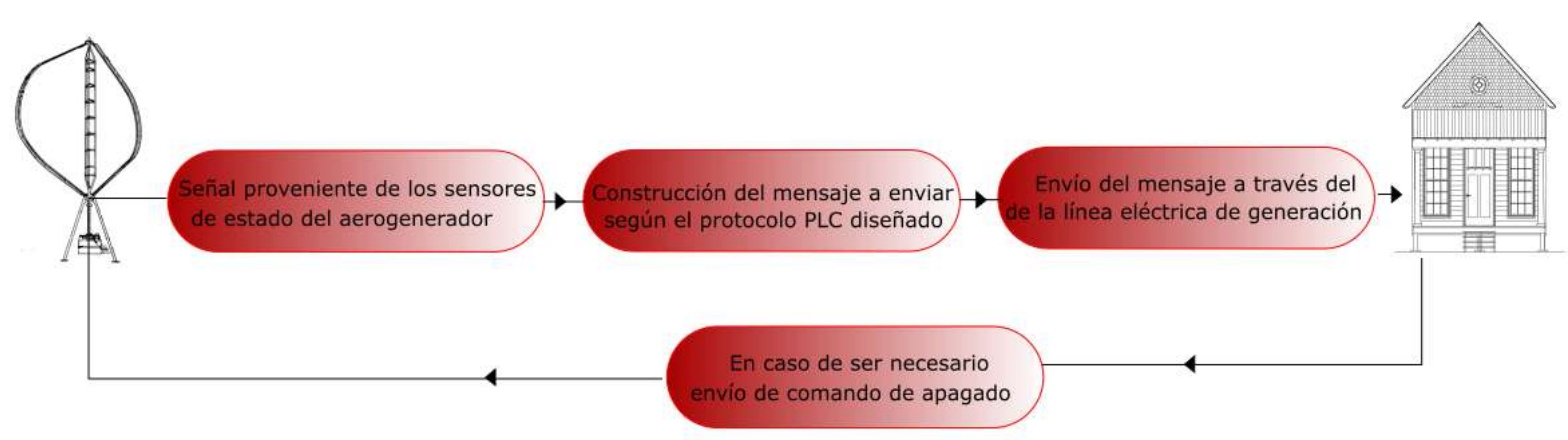

Figura 1: comunicación tipo PLC entre un aerogenerador de eje vertical y una vivienda/centro de control

A partir del estudio previamente mencionado, se comenzó una búsqueda bibliográfica puntual acerca de protocolos o estándares asociados a PLC que ya sean de público uso y conocimiento. Respecto a esto, se encontró una variedad muy amplia, pudiendo los mismos ser clasificados en tres grandes grupos: protocolos y técnicas en estándar para la realización de comunicaciones PLC Broadband (banda ancha), protocolos de comunicación para PLC Narrowband (banda estrecha) y una clase protocolos específicamente orientados a Smart Grid.

Si bien la aplicación a realizar en este plan de tesis es orientada a PLC Narrowband, no se descartó el estudio de protocolos y técnicas utilizadas en PLC Broadband. Sin embargo, tanto el rango de frecuencias que estos protocolos sugieren, sumado a técnicas de seguridad, que en el caso propuesto no son de tanto interés, acabaron siendo descartadas de todas formas.

Puntualmente se ponderó el tipo de modulación sugerida en cada caso, los cuales en su mayoría resultaron ser cuatro:

- $\quad$ DSSSM (Direct Sequence Spread Spectrum Modulation)

- $\quad$ OFDM (Orthogonal Frequency Division Multiplex)

- $\quad$ DPSK (Differential Phase Shift Keying)

- $\quad$ BPSK (Binary Frequency-shift keying)

Considerando que las líneas de transmisión de energía no fueron concebidas específicamente para el envío y recepción de datos, junto con la modulación los diferentes protocolos y estándares proponen diversas técnicas para mitigar el ruido en los canales de transmisión y recepción de información, para poder asegurar la integridad del mensaje. En particular en cada caso, se busca garantizar una buena relación señal ruido (SNR) y baja tasa de error de bit (Bit Error Rate). Algunas de las técnicas de procesamiento de señales que el estudio arrojó como las más utilizadas son:

Multicarrier Code División Multiple Access (MC-CDMA)

Multiuser detection (MUD)

Turbo Decoding 
- $\quad$ Dual-Hop Decode and Forward (Dual-Hop DF)

- $\quad$ Multi-Hop Routing

El ruido impulsivo (Impulsive Noise) es uno de los grandes problemas que presenta la técnica PLC, para mitigar el efecto del mismo durante la transmisión de datos, existen diferentes técnicas que se proponen, las más utilizadas se muestran a continuación:

- $\quad$ Structured compress sensing theory (SCS)

- $\quad$ Time Domain Suppression Schene

- $\quad$ Cyclic Redundancy Check (CRC)

- $\quad$ Combinación de diferentes preprocesadores no lineales (Linear Combining of Nonlinear Preprocessors)

\section{Resultados}

De los protocolos y estándares encontrados en diferente bibliografía, se han seleccionado aquellos que pueden llegar a ser compatibles con la comunicación tipo PLC bidireccional entre un aerogenerador que funciona bajo condiciones climáticas severas y una vivienda, la cual es alimentada por el mencionado aerogenerador. Considerando que el mensaje a enviar debe contener la respuesta de todos los sensores colocados en el aerogenerador para tener conocimiento del estado estructural del mismo, se consideró una determinada extensión de mensaje para ayudar a la selección de protocolos.

Como resultado final se redujo el número de protocolos o estándares posibles a tres, todos orientados a Narrowband PLC, capaces de ser aplicados en la situación propuesta.

Los próximos pasos a seguir son evaluar mediante simulación cuál de los protocolos seleccionados otorga una mejor performance, para ser puesto a prueba en un ámbito de laboratorio.

En este momento se está evaluando el software a utilizar para realizar la simulación completa ya que algunos de los protocolos que abarcan tanto la capa física como la capa de Acceso de Control al Medio (MAC) del Modelo de Interconexión de Sistemas Abierto (OSI). De acuerdo a lo que se ha analizado y en base a las referencias estudiadas, se concluye que se va a necesitar más de un 'software' para completar el análisis y estudio que este trabajo de tesis requiere.

\section{Referencias}

Antoniali, M., Tonello, A. M., Lenardon, M., \& Qualizza, A. (2011). Measurements and analysis of PLC channels in a cruise ship. 2011 IEEE International Symposium on Power Line Communications and Its Applications (págs. 102107). Udine: IEEE.

Artale, G., Cataliotti, A., Cosentino, V., Cara, D. D., Fiorelli, R., Russotto, P., \& Tinè, G. (2013). Medium Voltage Smart Grid: Experimental Analysis of Secondary Substation Narrow Band Power Line Communication. IEEE Transactions on Instrumentation and Measurement, vol. 62, no. 9, 2391-2398.

Bahamonde, P. J., Samela, A. M., Szewzuk, O., \& Ibarreta Fañanas, A. L. (2013). Estudio del Régimen de Vientos en la Localidad de Gobernador Gregores. Acta de la XXXVI Reunión de Trabajo de la Asociación, 131-137.

Choi, H.-J., \& Jung, J.-H. (2017). Enhanced Power Line Communication Strategy for DC Microgrids Using Switching Frequency Modulation of Power Converters. IEEE Transactions on Power Electronics, vol. 32, no. 6, 4140-4144.

Dégardin, V., Simon, E., Morelle, M., Liénard, M., Degauque, P., Junqua, I., \& Bertuol, S. (2010). On the possibility of using PLC in aircraft. ISPLC2010 (págs. 337-340). Río de Janeiro: IEEE.

Han, J., Jeong, J.-D., Lee, I., \& Kim, S.-H. (2017). Low-cost monitoring of photovoltaic systems at panel level in residential homes based on power line communication. IEEE Transactions on Consumer Electronics, vol. 63, no. 4, 435-441. 
López, G., Matanza, J., Vega, D. D., Castro, M., Arrinda, A., Moreno, J. I., \& Sendin, A. (2019). The Role of Power Line Communications in the Smart Grid Revisited: Applications, Challenges, and Research Initiatives. IEEE Access, vol. 7, 117346-117368.

Milioudis, A. N., Andreou, G. T., \& Labridis, D. P. (2015). Detection and Location of High Impedance Faults in Multiconductor Overhead Distribution Lines Using Power Line Communication Devices. IEEE Transactions on Smart Grid, vol. 6, no. 2, 894-902.

Prasad, G., \& Lampe, L. (2020). Full-Duplex Power Line Communications: Design and Applications from Multimedia to Smart Grid. IEEE Communications Magazine, vol. 58, no. 2, 106-112.

Rabie, K. M., Adebisi, B., \& Tonello, A. M. (2018). For More Energy-Efficient Dual-Hop DF Relaying Power-Line Communication Systems. IEEE Systems Journal vol. 12, no. 2, 2005-2016.

Takanashi, M., Takahashi, A., Tanaka, H., Hayashi, H., Harada, T., \& Hattori, Y. (2014). Channel measurement and modeling of high-voltage power line communication in a hybrid vehicle. 18th IEEE International Symposium on Power Line Communications and Its Applications (págs. 52-57). Glasgow: IEEE.

Tonello, A. M., Versolatto, F., \& Pittolo, A. (2014). In-Home Power Line Communication Channel: Statistical Characterization. IEEE Transactions on Communications, vol. 62, no. 6, 2096-2106.

Xu, Z., Yang, C., Tan, Z., \& Sheng, Z. (2017). Raptor Code-Enabled Reliable Data Transmission for In-Vehicle Power Line Communication Systems With Impulsive Noise. IEEE Communications Letters, vol. 21, no. 10, 2154-2157.

Yoon, S.-G., Jang, S., Kim, Y.-H., \& Bahk, S. (2014). Opportunistic Routing for Smart Grid With Power Line Communication Access Networks. IEEE Transactions on Smart Grid, vol. 5, no. 1, 303-311.

Zhilenkov, A. A., Gilyazov, D. D., Matveev, I. I., \& Krishtal, Y. V. (2017). Power line communication technologies in automated control systems. IEEE Conference of Russian Young Researchers in Electrical and Electronic Engineering (EIConRus) (págs. 246-249). St. Petersburg: IEEE. 